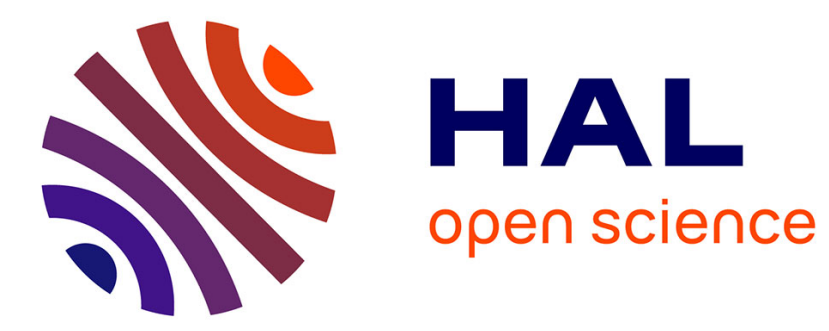

\title{
Les interprétations sémantiques de la prédication seconde intégrée: les relatives prédicatives
}

Claude Muller

\section{To cite this version:}

Claude Muller. Les interprétations sémantiques de la prédication seconde intégrée: les relatives prédicatives. Langue française, 2011, 171, pp.106-116. halshs-00989437

\section{HAL Id: halshs-00989437 https://shs.hal.science/halshs-00989437}

Submitted on 11 May 2014

HAL is a multi-disciplinary open access archive for the deposit and dissemination of scientific research documents, whether they are published or not. The documents may come from teaching and research institutions in France or abroad, or from public or private research centers.
L'archive ouverte pluridisciplinaire HAL, est destinée au dépôt et à la diffusion de documents scientifiques de niveau recherche, publiés ou non, émanant des établissements d'enseignement et de recherche français ou étrangers, des laboratoires publics ou privés. 
Les interprétations sémantiques de la prédication seconde intégrée: les relatives prédicatives ${ }^{1}$. Claude MULLER

Bordeaux-3 \& CNRS, UMR5610.

1. La construction des relatives prédicatives : verbes de perception et de mise en contact.

Les relatives prédicatives ${ }^{2}$ (pour une présentation récente et un bilan, cf. Furukawa, 2005, 2e partie) se distinguent des relatives ordinaires, appositives ou restrictives, par leur double aspect de constructions à la fois à incidence nominale (comme toutes les relatives, elles disent quelque chose d'un antécédent) et verbale: elles ont une relation au verbe qui varie, du plus au moins intégré, du statut de second complément direct (un 'objet' en termes de rôles sémantiques) pour les verbes de perception, à celui d'un complément temporel ou de manière. On s'intéressera dans ce travail à celles qui sont "à statut sémantiquement intégré" selon les propos de Cadiot \& Furukawa, 2000, caractérisant une des formes de la prédication seconde qui la distingue des expansions libres comme les appositions, quelquefois qualifiées de prédications "secondaires" plutôt que "secondes" (Melis 1988). Les constructions qui nous intéressent sont représentées typiquement par l'énoncé suivant :

(1) Ce prisonnier, je l'observe qui est assis ou couché comme défait et dévêtu de tout désir. (Saint-Exupéry, Citadelle, 1944, 765) ${ }^{3}$

Dans ces constructions, la prédication «seconde » est normalement le focus de la phrase, et donc est bien différente à la fois des relatives à caratérisation purement nominale, et des constructions apposées introduisant une incidente plus ou moins parenthétique. Elles ne sont pas librement construites sans une relation particulière avec un verbe. Sans cette relation, la construction est impossible :

(2) *On me l'a recommandé qui fera le travail

Leur intégration à la sémantique de la complémentation verbale est telle qu'on a parfois proposé (par exemple Schwarze, 1974) d'en faire une variante de complétive. Cette solution est partiellement inadéquate puisque ne pouvant rendre compte de nombreux exemples ayant des verbes sans construction complétive:

(3) Paul l'a photographiée qui faisait du ski / *Paul a photographié qu'elle faisait du ski.

Par contre, l'hypothèse d'une incidence double (Herslund, ce numéro) rend mieux compte des propriétés hybrides de ces relatives, à la fois liées lâchement à un antécédent, et d'une certaine façon compléments du verbe.

Il importe à cet égard de les distinguer d'une autre construction attributive, celle dans laquelle c'est le nexus entier qui occupe la fonction actancielle d'objet :

(4) Je crois Paul malade (= je crois que Paul est malade) construction qui n'a vraiment pas d'expression avec une relative prédicative ${ }^{4}$ :

(4') *Je crois Paul qui est malade

\footnotetext{
${ }^{1}$ Ce texte est la version revue et enrichie d'une communication inédite faite au colloque RSL II de Bruxelles (2005).

${ }^{2}$ Ou encore attributives (ce numéro, M. Herslund), « déictiques » pour Cadiot 1976.

${ }^{3}$ Sauf mention contraire, les exemples datés proviennent de Frantext.

${ }^{4}$ Une appositive est possible bien sûr: Je crois Paul, qui est malade. Mais alors, l'antécédent n'est pas pronominalisé : *Je le crois qui est malade.
} 
Dans Muller, 1995, j'ai proposé à la suite d'une hypothèse de van der Auwera, 1985, 1993, que pour les verbes de perception au sens strict, comme voir ou entendre, (et non pour des verbes du type de croire comme en (4)), le rôle sémantique "objet" puisse se dédoubler ou s'élargir. Contrairement à ce que proposait van der Auwera, je suppose cependant que dans ce cas la construction des compléments ${ }^{5}$ ne se confond pas avec le type "small clause", parce que l'objet (en tant qu'argument) du verbe principal n'est pas le nexus complet, mais un des deux termes (c'est aussi ce que dit Furukawa 2005, p. 104). Ainsi, avec un verbe de perception, la complémentation nominale seule est généralement possible avec un sens proche : Je vois Marie qui court / Je vois Marie, alors que les verbes 'de croyance' n'autorisent pas cette réduction : Je crois Paul malade $\neq$ Je crois Paul.

Dans le cas canonique des verbes de perception (c'est le type de verbe qui donne le plus lieu à la construction de ces relatives), la cible de la transitivité du verbe n'est pas le nexus propositionnel, qui figure syntaxiquement disjoint en (4) puisque les deux compléments sont séparables, mais qui est en totalité l'objet de croire. Avec les verbes de perception, la perception saisit soit le phénomène sensoriel, soit son agent ${ }^{6}$, et on peut alors admettre que la peception de l'action puisse sétendre à celle de son agent, ou vice versa.

Le fonctionnement syntaxique est celui des expansions attributives (comme le suppose aussi Herslund), l'antécédent étant le «sujet sémantique $»^{7}$ de la relative, qui équivaut à un participe présent : une prédication de syntagme verbal sans sujet lexical propre. En effet, le qui, sujet syntaxique en subordonnée, n'est ici que la réalisation du complémenteur rendu obligatoire par la flexion verbale conjuguée et dépendante (cf. Muller 2002). On le sait, ces relatives n'ont que cet introducteur, qui n'est pas le pronom «humain » homonyme. Cet introducteur est en relation de coréférence obligatoire avec un antécédent dans la principale. En somme, la relative dont l'introducteur est «qui » est sentie et utilisée comme une prédication verbale sans sujet sémantique, une sorte d'adjectif verbal à verbe conjugué et complémenteur, forme prédicative qui peut s'attacher à un "antécédent» sans s'y intégrer syntaxiquement, à la différence des relatives adnominales habituelles.

L'autonomie des deux constituants est telle que dans les constructions à verbe de perception, la perception peut cibler l'action verbale aussi bien que l'agent de l'action, comme le montre par exemple D. Leeman (2001) dans l'exemple ci-dessous ${ }^{8}$ à propos de la relative:

(5) Je vois les syndicats qui s'inquiètent

$=\quad$ Je vois l'inquiétude des syndicats / je vois les syndicats s'inquiéter

$\neq \quad$ Je vois les syndicats (D. Leeman, $2001: 378$ )

\footnotetext{
${ }^{5}$ Aussi bien un objet et une relative qu'un objet et un infinitif : je vois Marie courir /...qui court.

${ }^{6}$ La perception sensorielle sans l'agent est possible avec l'infinitif et certains verbes : J'entends crier chez les voisins. Avec une relative, le nom est obligatoire parce qu'antécédent, mais la perception est parfois aussi plutôt celle de l'action que celle de son agent: J'ai entendu quelqu'un qui criait, sans voir qui c'était. Cf. (5) cidessous.

${ }^{7}$ Il s'agit du sujet de prédication seconde, qui n'est pas nécessairement l'agent (par exemple si la relative a un verbe passif) et ne se confond évidemment pas avec un sujet syntaxique.

${ }^{8}$ Pour Prebensen 1982, les verbes de perception de ces constructions sont trivalents, avec une position d'actant locatif. Mais il ne semble pas adéquat de décrire systématiquement les relatives prédicatives comme des locatifs, et d'ailleurs la présence simultanée d'un locatif et d'une relative est possible : Emma l'aperçut dans la prairie, qui marchait sous les peupliers (Flaubert, cité par Prebensen, p.113). La construction disloquée de ces relatives, a fonction d'expansion facultative, semble un peu différente des constructions « intégrées » qui nous intéressent: le verbe principal ayant un autre complément, ces constructions semblent des circonstancielles assez lâchement rattachées à la principale : Il les regarda disparaître sous l'ombrelle, qui se retournèrent une ou deux fois (Aragon, Les beaux quartiers, Poche, 133). Comme on le voit ici, la position de second « patient » de regarda est déjà occupée.
} 
Cependant, l'extension de la construction à bien d'autres verbes, qui ne sont pas des verbes de perception sensorielle, ne permet pas de généraliser cette explication par le dédoublement du rôle actanciel d'objet. Ainsi, N. Furukawa (2005 : chapitre V) a joint à l'étude des relatives de verbes de perception celle de constructions proches produites par de nombreux verbes d'action' ${ }^{9}$ :

(6) Il l'a attrapée qui s'enfuyait dans la rue (N. Furukawa, $2005: 87$ )

Il propose d'y voir, avec les constructions plus étudiées de verbes de perception, une seule catégorie, les constructions "à deux événements amalgamés ». Le mode de contact des événements diffère, dans le premier cas, il s'agit de la perception / monstration d'un événement, dans le second d'un «amalgame » de deux événements lorsque le verbe permet l'interprétation d'un contact.

Ces dernières constructions à verbe d'action sont le fait de verbes sans subordination complétive, elles sont donc assez différentes des précédentes. Ainsi, l'hypothèse assez plausible d'un élargissement valenciel à deux objets perçus, justifiée dans le premier cas par la double complémentation directe, nominale et infinitive :

(7) Je l'ai entendu qui sortait / je l'ai entendu sortir / j'ai entendu sortir

ne se justifie pas dans le second cas. Avec un verbe d'action, le seul complément naturel est le complément nominal :

(8) On l'a attrapé qui sortait de chez vous avec votre portable

$=\quad$ On l'a attrapé

*On a attrapé sa sortie de chez vous

Cela ne remet cependant pas en cause le modèle syntaxique proposé ci-dessus : celui d'une relative qui a le statut fonctionnel d'un attribut de l'objet, à la manière d'un participe présent :

(8') On l'a attrapé sortant de chez vous avec votre portable

mais nous oblige à préciser les conditions dans lesquelles la greffe d'une telle relative est rendue possible avec ces verbes, alors qu'elle ne l'est pas pour d'autres. Il n'est pas question ici de supposer une valence extensible à deux compléments pour un rôle unique d'objet perçu. La seule explication tient à la relation de complémentation exercée par la relative à l'égard du verbe. Comme tout attribut de l'objet, la relative est alors en plus de sa fonction de prédicat de son antécédent, un complément du verbe principal. L'adjonction de ces constructions n'est pas libre, ainsi que l'illustrent (2) ci-dessus et (9), (10) :

(9) *Je l'ai conduit à la gare qui avait un train à prendre

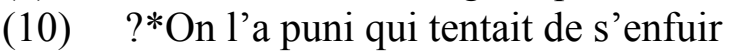

L'exemple (9) montre que la simple concomitance temporelle des actions ne suffit pas à justifier la relative ; on voit aussi en (10) qu'une éventuelle relation causale : parce qu'il tentait de s'enfuir, n'est pas non plus tolérée. On peut se demander si l'adjonction est régie par les possibilités d'extension de la valence verbale ${ }^{10}$ (ce qu'a également proposé en son temps Myra Rothenberg, 1979, qui voit dans les relatives prédicatives des "compléments facultatifs" et non des "compléments libres"). N. Furukawa (2005) suppose des constructions à expansion libre, mais soumises à des contraintes sémantiques, ce qui revient sans doute à dire autrement la même chose. Il faut que le verbe autorise la relative, et cela tient sans nul doute à la sémantique intrinsèque du verbe. Si on examine ces constructions (mais on verra qu'il en

\footnotetext{
${ }^{9}$ Cf. aussi Muller 2008 .

${ }^{10}$ Cette position est aussi celle de Lambrecht (2000: 50): La valence se trouve augmentée d'un terme.
} 
existe d'autres qui n'entrent pas dans ce cadre), on constate que les verbes autorisant la relative prédicative décrivent une action qui peut s'interpréter comme un mode de perception de l'action de son objet quand cet objet est saisi dans une "phase » de son action, comme dans cet énoncé :

(11) Ce matin, il l'a trouvée qui lui barrait le passage. Il ne la reconnaissait plus. Ce n'était plus la même femme...blogspot.com/2010/09.

Cet emploi de «trouver» n'est plus tout à fait celui du verbe à un seul objet direct : il est devenu le support d'une prédication "trouver quelque chose ainsi », sans perdre son interprétation matérielle (sans relever d'une analyse par complétive, trouver que quelque chose est ainsi, analyse inadéquate ici). Cela suppose que le verbe principal est un verbe de mise en contact avec l'objet dans un certain état ou en train d'accomplir une certaine action. Cette propriété de mise en contact, sensible dans trouver, attraper, n'est précisément pas pertinente dans les exemples non aceptables (9) et (10) : conduire, punir ne disent rien de l'absence ou de la présence antérieure à l'action du sujet sémantique de la relative. Les constructions décrites par N. Furukawa comme des 'constructions à deux événements amalgamés' sont en réalité des extensions de la catégorie des verbes de perception. On doit ajouter à ces verbes de mise en contact ceux qui décrivent un processus inverse, la cessation de contact, comme dans :

(12) Il l'a quittée qui faisait le ménage

Ces deux ensembles de constructions, verbes de perception et leurs extensions, et sous-classe de verbes d'action, ne forment pas cependant la totalité des constructions à relatives prédicatives, comme on va le voir maintenant.

\section{Les verbes régissant les relatives prédicatives}

Dans l'étude de cette construction par M. Rothenberg, 1979, se trouve une liste de verbes avec des exemples de cette construction, liste reprise par N. Furukawa 2005. A côté des deux constructions étudiées et signalées ci-dessus (verbes de perception / monstration, et verbes d'action à mise en contact avec le sujet sémantique), figure une sorte de résidu ("verbes divers") qui nous a amené à faire une recension aussi complète que possible des verbes pouvant de près ou de loin accepter une relative prédicative. Ce recensement doit être effectué dans des conditions qui évitent les interprétations restrictives-appositives habituelles: construction liée, interprétation non nominale du pronom "qui"11, antécédent sous la seule forme du pronom conjoint défini "le/les". En effet, la détermination qu'introduit la relative interfère avec la détermination du nom qui est son sujet sémantique : si la relative suit un groupe nominal indéfini, ou a pour antécédent un clitique indéfini comme en, elle peut s'interpréter comme une déterminative et les contraintes d'acceptabilité ne sont pas les mêmes. Par exemple, avec connaître:

(13) J'en ai connu qui n'ont pas hésité à renier publiquement le résultat de plusieurs années de labeur...(R. Martin du Gard, 1955, Souvenirs, 51)

Avec le défini, la phrase est à peu près inacceptable :

11 Le qui est le marqueur fonctionnel sujet, pas le pronom «humain » (analysable comme une variante de conjonction, cf. Muller 2002). Avec les verbes de perception, et un antécédent relatif, la construction bascule peut-être du côté des complétives, comme dans : La destinée qu'on sent qui s'accumule en silence (Montherlant, La reine morte, 1942, 152). Pour éviter cette interférence, je n'ai retenu que des constructions à antécédent pronom personnel. 
(13') ?*Je les ai connus qui n'ont pas hésité à renier publiquement le résultat de plusieurs années de labeur.

De même l'exemple (9) répété ci-dessous devient acceptable quand l'antécédent de la relative est le clitique indéfini en (9') remplaçant le défini le.

(9) *Je l'ai conduit à la gare qui avait un train à prendre

(9') J'en ai conduit à la gare qui avaient un train à prendre

L'emploi d'un clitique défini comme le exclut une interprétation usuelle : celle de relative déterminative, à valeur restrictive sur un ensemble indéfini. Il y aurait d'ailleurs de bonnes raisons de s'intéresser à ces restrictives en position focalisée, puisqu'elles sont à l'origine de constructions présentatives (comme il y en a qui...) mais leur fonctionnement n'est pas le même que celui des prédicatives, et leur distribution ne semble pas (ou pas au même degré) contrainte, comme le montre (9').

La classification des verbes permettant notre construction est donc celle des verbes transitifs à objet clitique défini en antécédent, avec des spécifications sémantiques: si on laisse de côté les constructions locatives, syntaxiquement différentes ${ }^{12}$, on trouve les grandes classes suivantes dans les études sur cette question:

1- verbes de perception (types "voir, entendre, sentir") et verbes de monstration qui sont des formes causatives de la perception "montrer, exposer). Cela inclut la perception mentale "imaginer").

2- verbes d'événement impliquant la perception (types: "trouver", attraper", inversement "quitter, laisser"). Le sujet du verbe principal agit de telle façon qu'il entre en contact avec le sujet sémantique de la relative pendant son action - les verbes inverses supposant un processus parallèle, le sujet principal abandonne le sujet sémantique à son activité.

3- les verbes "divers", qui n'entrent pas dans ces deux classes. Par exemple:

(14) On l'a choisi qui sortait de Polytechnique (M. Rothenberg)

(15) Je l'ai connu qui vendait des cravates sur le boulevard

J'ai examiné tous les verbes susceptibles de se construire avec une relative ayant pour antécédent le pronom conjoint défini. J'ai recensé près de 200 verbes ${ }^{13}$, l'acceptabilité des exemples étant plus ou moins bonne, suffisamment ${ }^{14}$ en tout cas à mon sens pour que la construction soit produite si besoin est. Cet examen m'a conduit à supposer que la construction est déterminée non seulement par la sémantique du verbe principal (puisque seul un sous-ensemble des verbes transitifs permet cette construction) mais aussi par la sémantique de la relative, puisque certains verbes principaux qui excluent cette construction pour certaines relatives semblent l'accepter pour d'autres (comme connaître dans l'exemple (15) ci-dessus, à comparer avec la phrase (13’) à peu près inacceptable citée auparavant). La construction canonique (verbes de perception) comporte une relative à sens le plus souvent concret, événementiel, saisie dans son cours (la paraphrase "en train de + infinitif" est alors

\footnotetext{
${ }^{12}$ C'est le type : Il est là-bas qui rédige son rapport. Il a été étudié par Furukawa 2005 (ch. 4). Il y a aussi des constructions à objet clitique antécédent et locatif, dans lesquelles la relative prédicative semble moins liée à la construction verbale. Nous avons laissé de côté des constructions de ce type, exemplifiées par les exemples suivants de Prebensen : Elle le laisse dehors qui sanglote au jardin. Ou encore : Quand nous l'eûmes là, devant nous, qui remplissait de sa splendeur cette misérable chambre,...(Prebensen, p. 113). Les relatives sont plus nettement des adjonctions facultatives équivalentes à des participes présents.

${ }^{13}$ La liste de M. Rothenberg comporte 71 verbes de cette construction (antécédent pronom conjoint défini objet direct). Notre annexe propose une liste de 193 verbes.

${ }^{14}$ Contrairement à ce que dit Prebensen, les jugements d'acceptabilité de M. Rothenberg ne me semblent généralement pas sujets à caution. Certains, qui sont un peu marginaux, sont notés ? dans la liste.
} 
adéquate du point de vue aspectuel). C'est aussi l'interprétation de la relative avec des verbes d'action comme trouver, surprendre. Mais une autre classe de constructions est possible, avec une relative signifiant cette fois une propriété plutôt qu'un événement en cours. On peut ainsi comparer l'exemple (14) de Rothenberg (une propriété) avec la construction, soit perceptive, soit d'événement simultané, (16):

(14) On l'a choisi qui sortait de Polytechnique

et:

(16) On l'a vu qui sortait de Polytechnique / On l'a rencontré qui sortait de Polytechnique

Le sens perceptif est bien distinct du sens de propriété "sortir de Polytechnique" qui apparait avec choisir : "sortir de Polytechnique », en tant que propriété, c'est «avoir fait ses études à Polytechnique ». Il suffit d'ajouter "en courant" ou "rapidement" pour exclure le sens de propriété et revenir au sens d'événement en cours (ce qui rendrait inacceptable la construction avec choisir, sauf jeu de mots). De nombreuses relatives au sens de "propriété" peuvent se construire ainsi. Le sens est alors analogue à celui des attributs adjectivaux, et implique probablement la transitivité verbale à la manière des prédications secondes adjectivales (c'est le sens "sélectionnel" des constructions attributives de Muller, 2000, terme et sens définis ainsi pour les adjectifs en prédication seconde par K. Olsson-Jonasson 1976).

Pour cette interprétation de propriété attachée à des formes verbales, on peut se référer à $\mathrm{M}$. Riegel, 1985 (205): «Les formes proprement verbales oscillent entre une lecture événementielle et une lecture stative ». Il appelle cette dernière interprétation une "propriété dérivée" ontologiquement seconde. Un certain nombre de relatives construites sur ce modèle sont ainsi des propriétés, et font ressortir par contraste la particularité des constructions courantes de ce type liées à la perception, constructions qui sont bien des événements. L'interprétation "propriété" n'est pas impossible avec les verbes de perception, mais elle est secondaire et marginale dans ce cas, sauf emploi non événementiel (au sens d'événement concret):

(17) Je le vois encore qui sortait de Polytechnique, promis à un brillant avenir

(dans la forme indirecte liée à la perception d'un souvenir; le sens événementiel n'est plus obligatoire). La subordination facilite aussi le sens non événementiel:

(17') Quand je les vois qui sortent de Polytechnique et qui manquent totalement de sens commun, je suis amené à juger sévèrement de la formation de nos ingénieurs

Une catégorie particulière du type "propriété" est celle qui s'attache à un antécédent général, la relative, souvent au présent, décrivant une qualification durable, quasi générique sur le sousensemble:

(18) Ces montres, on les fournit qui résistent à 10 atmosphères

Une autre catégorie de verbes avec une relative au sens de "propriété" s'attache à des antécédents particuliers. La relative décrit alors un état représentant un stade variable dans le développement de l'antécédent (état transitoire). Par exemple:

(19) Je l'ai connu qui vendait des cravates sur les boulevards, et maintenant il dirige une grande entreprise!

On a distingué cette classe de constructions de la précédente: la relative n'est pas généralisable à une classe d'individus, elle décrit de plus un état transitoire et comporte souvent des indications aspectuelles, par exemple:

(20) On l'a accepté qui marchait encore à peine (M. Rothenberg)

Les deux classes "propriété" qu'on a retenues pour les relatives correspondent par conséquent à une interprétation de complément adverbial du verbe schématisable grossièrement par "dans 
un certain état" ${ }^{15}$, l'une supposant une généralisation, l'autre étant liée à la caractérisation transitoire d'un objet unique. A la différence des classes «événements », les «propriétés » sont une sorte de réanalyse des actions en caractérisations de manière, la frontière n'étant pas toujours nette.

Il va de soi que ces deux grandes catégories sémantiques de relatives existent aussi dans les constructions adnominales habituelles. Simplement, dans ce cas, la différence entre une opération de sous-catégorisation de l'antécédent par une action ou par une propriété n'a pas d'effet majeur sur l'interprétation : l'homme qui sort de Polytechnique peut aussi bien, selon les cas, distinguer un ingénieur polytechnicien ou un individu surpris en train de sortir matériellement et physiquement de cette institution.

\section{Les catégories.}

Les constructions peuvent être classées en deux grandes catégories, subdivisables elles-mêmes en deux sous-classes qui sont parfois très proches pour certains exemples pour lesquels on peut hésiter entre deux constructions. Si on se contente de noter pour chaque verbe la (parfois les ${ }^{16}$ ) construction préférentielle de la relative, on obtient les résultats suivants:

Classe A: Verbes de perception / monstration, la relative ayant un sens événementiel (incluant un état particulier): 46 verbes (incluant voici). Statistiquement, c'est massivement l'emploi le plus fréquent, ce qui explique qu'on l'ait parfois exclusivement retenu pour définir ces constructions ("compte rendu de perception" pour les relatives de ce type ${ }^{17}$ ). Un petit sousensemble peut sélectionner indifféremment l'action ou son agent, ce qui se traduit par une possibilité de construction infinitive ${ }^{18}$ pour une action sans sujet mentionné :

(21) J'entends couper du bois dans la cour / je les entends couper du bois dans la cour

(22) Je les entends qui coupent du bois dans la cour.

Classe B: Verbes d'action sans construction complétive, saisissant leur objet en train d'agir. La sémantique de la relative est du même type que dans la classe A: 75 verbes, beaucoup d'emplois relativement marginaux mais d'une bonne acceptabilité :

(23) Marie l'a croisé qui rentrait de sa partie de tennis.

Classe C: Verbe d'action ou de représentation (savoir) saisissant l'objet dans un état transitoire (une propriété non définitive, en tout cas pas un événement concret isolé) sans généralisation à une classe (les verbes d'action sont interprétés ici comme symptômatiques de l'état de leur agent) : 51 verbes.

(24) Je l'ai connu qui marchait à peine!

\footnotetext{
15 Avec les verbes de perception ou d'action, l'interprétation hors contexte de la phrase est celle d'une caractérisation par prédication événementielle mais l'interprétation "propriété »n'est pas exclue si le contexte s'y prête: Je l'ai rencontré cet été qui sortait tout juste de Polytechnique, il venait d'avoir son diplôme.

${ }_{17}^{16}$ On obtient ainsi 202 constructions pour 193 verbes.

17 Terme de G. Kleiber 1988, repris par Lambrecht 2000. Ce dernier admet encore de nombreuses restrictions posées initialement dans G. Kleiber 1988, restrictions qui sont pour certaines excessives (Muller 1995, voir également Leeman 2001). On a trouvé facilement, dans un corpus assez vaste, des exemples avec des pronoms de 1ère/2ème personne, avec d'autres modes que l'indicatif (plus de 100 exemples à l'imperatif), avec le futur, avec la négation, avec des pronoms réfléchis (contrairement aussi à ce qui dit Rothenberg 1979, 379). On a noté de tels exemples dans la liste fournie.

${ }^{18}$ La réalisation comme complétive finie n'est pas toujours possible avec les verbes de perception : J'écoute la pluie qui tombe, j'écoute pleuvoir, *j'écoute qu'il pleut.
} 
Classe D: Verbes construits le plus facilement avec un sens général ou habituel, portant sur une classe d'objets auxquels est attribuée une propriété durable: 30 verbes.

(25) Ces montres, on les construit qui résistent à une pressions de 10 atmosphères.

Il est à noter que tous ces emplois sont illustrés par quelques exemples de M. Rothenberg, même si cette dernière n'a pas reconnu ce qu'on a isolé en tant que classes $\mathrm{C}$ et $\mathrm{D}$ et n'en parle pas. Les recherches sur corpus sont assez décevantes, même si quelques exemples illustrent les classes $\mathrm{C}$ et $\mathrm{D}$. Massivement, les corpus contiennent des constructions des classes A et dans une moindre mesure (mais avec une plus grande variété de verbes) B. Comme les participes présents, dont elles sont proches, les relatives en qui ont des propriétés verbales et des propriétés plutôt de type adjectival et de caractérisation. Il est clair que les verbes des classes A et B sélectionnent des relatives prédicatives ayant des propriétés verbales, avec la sémantique qui s'y attache, d'action ou d'état en cours de développement. Inversement, les verbes des classes $\mathrm{C}$ et $\mathrm{D}$ sélectionnent de préférence des relatives prédicatives à propriétés de type adjectival, à fonction d'attribut ou de circonstant. Les différences importantes de fréquence d'utilisation entre les classes pourraient tenir à la plus ou moins grande intégration à la valence verbale, d'une part, et à la possibilité d'interpréter naturellement la relative comme un équivalent d'adjectif, d'autre part. Ainsi, la grande fréquence de constructions du type A pour les verbes de perception les plus courants est probablement liée à l'élargissement valenciel possible du rôle sémantique « objet» à deux termes, action et agent. Les verbes de la classe B ont comme actant naturel l'antécédent, et la relative se construit comme une prédication seconde de type circonstanciel « en train de... », mais on remarquera que le sens n'est pas tout à fait le même avec la relative: la transitivité a tendance à inclure l'action dans la portée sémantique donc dans l'interprétation, du verbe principal, et ces verbes (par exemple surprendre, trouver) sont dans la continuité sémantique de verbes de perception: par exemple, trouver quelque chose devient trouver quelque chose ainsi. Ils ont tendance dans ces constructions à prendre une interprétation particulière tenant à l'intégration de la relative dans la complémentation :

(26) Il a quatre-vingts ans sonnés et je l'ai trouvé qui arrachait des genêts sur la côte...(H. Pourrat, Gaspard des montagnes, 1931, 31-32).

Ce qui rassemble ces deux classes, c'est l'interprétation événementielle de la relative. Par contre, pour les classes $\mathrm{C}$ et $\mathrm{D}$, la prédication relative prend le sens d'une "propriété »: même avec un verbe d'action, c'est une interprétation dérivée qui s'impose : soit un statut acquis par une action habituelle (comme « vendre des cravates » en (15) : la propriété associée est celle d' "être vendeur de cravates »), ou un sens non actif du verbe : "sortir d'une grande école » : l'action devient une caractéristique de son actant, et non un événement épisodique. L'interprétation est similaire à ce que serait la réalisation d'un adjectif dans cette construction. Le lien à la valence verbale est à rattacher à l'analyse plus générale que l'on fait des attributs de l'objet ${ }^{19}$. Le sens "sélectionnel" qu'on a dégagé dans la construction adjectivale (Muller, 2000) correspond bien en tout cas à la classe D, qui est une seconde caractérisation opérant sur du défini. La classe $\mathrm{C}$, qui est celle des états transitoires, est également typique des constructions adjectivales. Dans les énoncés suivants, l'interprétation de l'attribut, qu'il soit adjectif ou relative, est exactement du même type :

(15) Je l'ai connu qui vendait des cravates sur les boulevards (...vendeur de cravates)

\footnotetext{
${ }^{19}$ Pour mémoire, la relative (attribut) et son antécédent sont tous deux et dans tous les cas des compléments syntaxiques distincts du verbe. Les variations entre constructions, parfois avec le même verbe, tiennent à la structure d'arguments. Voir pour les adjectifs en prédication seconde Muller 2000.
} 


\section{Conclusion.}

On a essayé dans ce travail d'avoir une vision exhaustive des relatives "prédicatives » dans leur construction la plus représentative, lorsque leur apport se fait sur un terme déjà défini. A côté des constructions les plus employées et les plus utilisées, les relatives «compte rendu de perception » (notre classe A), à quoi s'ajoute la catégorie des constructions à «mise en contact » entre deux actions (catégorie B), on a montré qu'il existait un autre type d'extension par relatives, sélectionnant d'autres verbes recteurs : la relative à interprétation de propriété plutôt que d'action. Dans ces constructions, plus marginales en proportion mais tout de même repérables, la relative est dans la distribution des adjectifs attributs en prédication seconde, et on ne sera pas surpris d'y trouver des sous-catégories analogues : la classe des propriétés ou états transitoires, liée à une interprétation de temps partagé ( $\mathrm{X}$ alors qu'il est dans l'état $\mathrm{Y}$ ), notre classe $\mathrm{C}$, et celle des propriétés sélectionnelles qui recatégorisent de façon plus restreinte un groupe nominal déjà défini (la classe D).

\section{Annexe: liste des constructions ${ }^{20}$.}

On l'a abandonné qui gisait sur un grabat (MR): B

On l'a abattu qui sortait de chez lui: $\quad$ B

On l'a accepté qui marchait encore à peine (MR) C

Je l'ai accompagné qui allait à son travail B

On l'a accostée qui attendait son bus $\quad$ B

Je les ai accueillis qui manquaient de tout $\quad$ C

Je les achète qui pèsent déjà $50 \mathrm{kgs}$. $\quad \mathrm{D}$

Je l'ai admiré qui soulevait une barrique de vin (MR) A

On l'a admise qui savait à peine lire (MR) C

Il l'a affronté qui voulait entrer de force B

On l'a aidé qui cherchait à s'installer à son compte $\quad \mathrm{C}$

Je l'aperçois qui entre $\quad$ A

Il les a apostrophés qui passaient sous ses fenêtres $\quad$ B

?(Les affiches) On les appose qui appellent à manifester demain $\quad$ D

On l'a appréhendé qui tentait de voler une voiture B

On l'a arrêté qui tentait de voler une voiture B B

On l'a arrosé qui commençait tout juste à sécher $\quad$ C

On l'a attaqué qui rentrait chez lui $\quad$ B

On l'a attrapé qui se cachait dans la cave B

Je l'avise un jour qui débouchait de sa caserne (Prebensen 1982, 115) A

$\mathrm{Au}$ XVIIIe siècle, toute le monde portait les cheveux longs : Louis XVI les avait qui tombaient jusqu'au bas du dos (www.passion-histoire.net, 18/04/09)

Sa maison, il l'a bâtie qui donne sur la rivière

On l'a blessé qui rentrait chez lui $\quad$ B

On l'a bloqué qui cherchait à s'enfuir $\quad$ B

On l'a bouclé qui clamait son innocence B

On l'a bousculé qui sortait de l'argent à un distributeur B B

On l'a braqué / buté/ qui... (idem) B B

?Je l'ai caché qui avait des poursuivants à ses trousses $\quad$ B

On l'a capturé qui se cachait dans une église $\quad$ B

\footnotetext{
${ }^{20}$ La notation MR signale un exemple de la liste de Rothenberg. Les lettres renvoient aux classes de relatives. On a parfois ajouté à la liste une exemple réel, soit avec la même classification, soit avec une classification différente.
} 
On l'a chahuté qui arrivait en retard

On l'a chassé qui cherchait à s'introduire dans la maison $\quad \mathrm{B}$

On l'a cherché qui errait dans le noir B B

On l'a choisi qui sortait de Polytechnique (MR) D D

On l'a chronométré qui courait un cent mètres (MR) B

Je les commande qui soient le plus frais possible $\quad$ D

On l'a complimenté qui avait rempli sa tâche à la perfection $\quad$ C

Les airs, je les compose qui s'accordent avec les paroles D

On les conçoit qui puissent s'adapter aux besoins à venir $\quad$ D

On les confectionne ... (idem) D

On l'a congédié qui prenait de l'argent dans la caisse $\quad$ C

Je l'ai connu qui vendait des cravates sur les boulevards $\quad$ C

$\begin{array}{ll}\text { Connais-le qui s'élève à travers toutes tes épaisses raisons. } & \\ \text { (Ch. Maurras, Le chemin de paradis, 1927, 86) } & \text { A }\end{array}$

Elle le considérait qui feuilletait un mémoire (MR) A

Je l'ai consolé qui pleurait la perte de son joujou $\quad$ C

On l'a construite qui résiste aux tremblements de terre D

Je le contemplais qui montait l'escalier (MR) A

Locomotive le contemple qui lisse ses ailes avec soin puis consulte sa kelton A

(J. Vautrin, Bloody Mary, 1979, 43)

On l'a contrôlé qui transportait des produits dopants B

On les côtoie qui font la fête, sans qu'on y participe $\quad$ B

On l'a coulé qui approchait dangereusement de la base B

On l'a couronné qui était encore un petit enfant $\quad$ C

Ce modèle, on l'a créé qui soit mettable par tous les temps D

On l'a critiqué qui soutenait sa théorie $\quad$ B

On l'a croisé qui rentrait chez lui B B

Les fleurs, on les a cueillies qui commençaient à se faner $\quad$ C

$\begin{array}{ll}\text { On l'a débarqué qui puait l'alcool B } & \text { B }\end{array}$

Je l'ai décelé dans le tas qui différait du reste (MR) C

On l'a découragé qui voulait faire l'ascension sans oxygène $\quad \mathrm{C}$

On l'a découvert qui dormait dans un coin de la pièce A

Toujours ces marques, toujours ces traces et toujours ces signes tu les découvriras qui découlent les uns des autres (Saint-Exupéry, Citadelle, 693)

On le décrit qui est toujours en forme

Il la décrit qui porte une robe voyante (MR)

$\mathrm{D}$

Je l'ai dégoté qui jouait à la belote dans le bistro du coin (MR) B

Je l'ai déniché qui chantait dans les foires (MR) B

On l'a dépassé / doublé qui roulait vers Nogent B

On l'a dépeint qui écrivait ses poèmes debout face à la mer $\quad \mathrm{C}$

Il le dépeint qui traverse la Seine à la nage (MR) B

On l'a désigné qui s'occuperait de l'intendance $\quad \mathrm{C}$

On l'a détaché qui avait du mal à respirer $\quad \mathrm{C}$

On l'a détruit qui s'approchait dangereusement de la ville B

Je le devinais qui surveillait mes notes (MR) A

Il l'avait deviné qui s'éloignait, descendait, capitulait... A

(P. Moinot, Le guetteur d'ombre, 1979, 306)

?On le dit qui est toujours partant pour une aventure ${ }^{21} \quad \mathrm{C}$

Je l'ai discerné au loin qui se dirigeait vers le bar (MR) A

Il l'a distinguée dans la foule qui portait une robe blanche (MR) A

Il l'a draguée qui traînait dans les boîtes de nuit $\quad$ B

Il l'a ébauché qui tenait son chien par la laisse (MR) C

${ }^{21}$ Jacques veut dire, qui renverse, qui supplante.(...) Or, on le dit qui renverse parce qu'il renversa le monde...(www.abbaye- saint-benoit.ch). C'est nettement une propriété (type D). L'emploi est marginal parce que la relative est pratiquement une citation à valeur métalinguistique. L'exemple inventé serait plutôt un état transitoire. 
La voiture l'a éclaboussé qui rentrait chez lui à pied

On l'a éconduit qui cherchait à se faire embaucher comme jardinier

Je l'ai écouté qui jouait du violon

On les a emballés qui étaient encore chauds

On l'a enchaîné qui se débattait encore

Je l'ai enduré qui n'arrêtait pas de pleurer (MR)

On les a enfermés qui n'avaient pas eu le temps de se changer

On l'entend qui ronfle

On les a entreposées qui étaient déjà bien mûres

Je l'ai entrevu qui se faufilait derrière la cuisine (MR)

On l'entrevoit qui va et vient (Genevoix, Les Eparges, 618)

Je l'ai épié qui volait dans un grand magasin (MR)

On l'a espionné qui cherchait à écouler des marchandises volées

On l'a éveillé qui parlait dans son sommeil

On les a évacués qui transportaient ce qu'ils avaient pu sauver

Il l'a évoqué qui jouait du violon (MR)

On les fabrique qui résistent aux chutes

On les fait qui résistent aux chutes

On l'a fait décamper/déguerpir qui était en train de tagger le mur

Je me le figure qui fait son numéro

Il l'a filmée qui escaladait le rocher

Il le flairait qui s'approchait en se dandinant (MR)

On l'a formé qui puisse s'adapter aux situations nouvelles

Je les fournis qui résistent aux chocs

On le fréquentait qui faisait encore ses études de droit à l'époque

On l'a fui qui cherchait à nous parler

Je la garantis qui résiste à tout

Il l'a giflée qui lui disait des insultes

Il l'a grondé qui arrachait les fleurs de ses jardinières

Je l'ai guetté qui allait descendre de sa chambre (MR)

Il le hèle qui sort de chez lui

Il l'a heurté qui sortait du porche

Il l'a identifié qui sortait du métro

Il l'a identifié qui traversait la place (MR)

Je l'imagine qui se peigne devant mon miroir (MR)

Tout le monde m'ignore qui suis à l'affût. (M. Tournier, Les Météores, 1975, 86)

Il l'imite qui fait son cours, c'est très amusant

On l'a immobilisé qui tentait de fuir

Il l'implorait qui s'avançait vers lui

Il l'a injurié qui passait simplement devant sa porte

$\mathrm{C}$

$\mathrm{C}$

$\mathrm{C}$

$\mathrm{C}$

A

$\mathrm{C}$

Il l'a insulté qui passait simplement devant sa porte

On l'a intercepté qui se déplaçait avec une bombe

On l'a interpellé qui avait brûlé un feu rouge

On l'a interrogé qui sortait de chez lui

On l'a interviewé qui sortait de son bain

On l'a joint qui sortait de son domicile

On l'a jugé qui pouvait à peine tenir debout

Je l'ai laissé qui faisait la vaisselle

Les frites, il les mange qui baignent dans l'huile $\quad$ D

On nous l'a montré qui faisait du jogging A

On l'a moulu qui n'était pas tout à fait sec, ce café $\quad$ C

On les a naturalisés qui ne parlent même pas le français $\quad \mathrm{C}$

\footnotetext{
${ }^{22}$ On l'a compté dans les verbes de monstration, comme le pendant causatif des verbes de représentation mentale.
} 
Je l'ai observé qui se glissait entre les tables (MR)

A

Ce prisonnier je l'observe qui est assis ou couché comme défait et dévêtu de tout désir (Saint-Exupéry, Citadelle, 765)

De cette façon, on les obtient qui sont toutes fraîches

A

On les pêche qui mesurent au moins $20 \mathrm{~cm}$, sinon on les remet à l'eau

Il l'a peinte qui tenait son enfant dans les bras (MR) A

Je le percevais qui commençait à s'inquiéter $\quad$ A

Il la percevait qui vibrait intérieurement (MR) A

On l'a photographié qui jouait au tennis $\quad$ A

On l'a pincé qui volait à l'étalage B

On l'a planté qui fleurissait déjà $\quad \mathrm{C}$

On l'a plié qui n'était pas encore sec, ce linge $\quad$ C

On l'a poursuivi qui s'enfuyait B

On l'a poussé qui marchait au bord du quai B

On les préfère qui sortent des grandes écoles $\quad$ D

?Je le pressentais qui allait fondre en larmes (MR) A

On l'a pris qui cachait des marchandises dans ses poches B

Le jour les prit qui se parlaient à mi-voix....Blanche consentit à s'étendre vers cinq heures du matin (Aragon, Les voyageurs de l'impériale, 1947, 296)

On l'a publié qui incluait le passage litigieux $\quad$ C

Il l'a quittée qui faisait le ménage B

Je l'ai raccompagné qui rentrait chez lui $\quad$ B

Je l'ai rachetée qui était presque neuve $\quad$ C

Je l'ai ramassé qui traînait sur le trottoir $\quad$ B

?Je l'ai rappelé qui avait oublié ses clés $(\mathrm{MR})^{23} \quad \mathrm{C}$

Je me le rappelle qui se levait tous les jours à six heures (MR) A

Je l'ai rattrapé qui marchait vers la rivière B

Je l'ai reçu qui revenait de donner une leçon particulière B

$\begin{array}{ll}\text { On les recherche qui pèsent plus de } 10 \mathrm{kgs} & \mathrm{D}\end{array}$

Je l'ai reconnu qui sortait du cinéma A

Je l'ai recruté qui sortait de Polytechnique D D

Je l'ai recueilli qui ne savait pas où aller $\quad \mathrm{C}$

$\begin{array}{ll}\text { Je l'ai récupéré qui traînait dans un placard } & \mathrm{C}\end{array}$

?Je la redoutais qui allait surgir de l'ombre (MR) A

Je l'ai regardé qui travaillait $\quad$ A

Je l'ai rejoint qui marchait vers la station de métro B

On l'a relevé qui venait de se casser la jambe $\quad \mathrm{C}$

Je l'ai remarqué qui se glissait derrière vous (MR) B

Je l'ai rencontré qui allait au cinéma B

On nous l'a rendu qui n'était plus intact $\quad$ C

Il le représente qui ne peut s'empêcher de pleurer (MR) A

Je me le représente qui arbore une cravate neuve (MR) A

C'est ainsi que Vous nous portez et que nous Vous ressentons qui nous portez (P. Claudel, Commentaires et exégèses, 1948, 178)

On nous l'a restitué qui n'était plus intact

Je l'ai repéré qui cherchait à s'introduire chez vous

Je l'ai retrouvé qui jouait du saxo dans un bar

On les a réunis qui travaillent sur la même question $\quad$ D

On les a réveillés qui dormaient profondément $\quad B$

Il l'a révélée qui se déshabillait (MR) A

Je les revois encore qui faisaient leurs devoirs sur la table de la cuisine A

Il les saisit qui brûlent encore

A

C

A

$\mathrm{B} / \mathrm{C}^{24}$

B
A
A

\footnotetext{
${ }^{23}$ Acceptabilité pas très bonne. J'ai trouvé l'exemple suivant, qui ressemble cependant plus à une parenthétique : Quant au rôle spécifique du gouverneur, le ministre Clément Sawadogo l'a rappelé qui consiste à l'appropriation et au raffermissement de la bonne gouvernance...(lefaso.net, 2/10/2007)

${ }^{24} \mathrm{~B}$ s'il s'agit d'un événement fortuit, $\mathrm{C}$ si c'est une propriété (il est alors saxophoniste de bar).
} 
Il l'a salué qui sortait du tribunal

Qu'il la sache qui l'aime, et qu'il l'ait à sa seule volonté, ...

$\mathrm{D}$

(P. Claudel, Le soulier de satin, 1944, 2ème journée, sc5)

$\mathrm{C}$

Je l'ai senti qui bougeait (MR)

A

On les a secourus qui se croyaient déjà perdus $\quad \mathrm{C}$

On les sélectionne qui courent vite $\quad$ D

On me m'a signalé qui circulait à cette heure-là sur les quais $\quad \mathrm{A}$

On l'a souffert qui arrivait tous les jours en retard (MR) C

Je l'ai soutenu qui escaladait la grille

On l'a stoppé qui se dirigeait vers l'aéroport B

On l'a suivi qui se dirigeait vers l'aéroport $\quad$ B

Je les ai supportés qui pleuraient des heures entières (MR) $\quad$ C

$\begin{array}{ll}\text { On l'a surpris qui volait des pommes } & \mathrm{A} / \mathrm{B}\end{array}$

On les surveillait qui faisaient leurs devoirs $\quad$ A

On les taille qui ne dépassent pas le mur $\quad$ D

Il l'a trouvé qui buvait dans un bar $\quad$ B

On les utilise qui ont déjà servi $\quad$ D

On les vend qui pèsent au moins $10 \mathrm{kgs} \quad \mathrm{D}$

On les veut qui soient à même de répondre aux besoins $\quad \mathrm{D}$

Les sacs synthétiques sont très gros si on les veut qui résistent aux basses températures...(voyageforum.com, 19/09/2008)

Je le visualise qui s'assoit sur son lit pour pleurer (MR) A

Le voilà qui arrive $\mathrm{A}$

Je le vois qui revient du marché A

Je me vois qui vous regarde, et non point seulement ici et maintenant, mais demain et plus tard, dans mes palais, dans mes conseils, dans le tombeau (Audiberti, Théâtre, 1948, 171).

A

\section{Références bibliographiques}

AUWERA J. VAN DER, (1985), The predicative relatives of French perception verbs, in M.Bolkenstein e.a. (eds) : Predicates and Terms in Functional Grammar, Dordrecht: Foris, p. 219-237.

AUWERA J. VAN DER, 1993, Les relatives prédicatives du français, Travaux linguistiques du Cerlico, 6, Rennes-2, p. $265-277$.

CADIOT P., (1976), Relatives et infinitives déictiques en français, DRLAV, 13, p. 1-64.

CAdiot P. \& N. FuruKaWA, (2000), Présentation, Langue Française 127, p. 3-5.

FurukaWa N., (2005), Pour une sémantique des constructions grammaticales, , Bruxelles : De Boeck-Duculot.

GUIMIER E., 1998, Les constructions à prédicat de l'objet des verbes de perception, in M. Forsgren, K. Jonasson $\&$ H. Kronning (eds): Prédication, assertion, information, Actes du colloque d'Uppsala, Uppsala: Acta Univ. Upsaliensis, p. 231-241.

HERSLUND M. (ce numéro), La relative attributive comme cas d'hypotaxe complexe.

KLEIBER G., 1988, Sur les relatives du type je le vois qui arrive, Travaux de Linguistique, 17, p. 89-115.

LAMBRECHT K., 2000, Prédication seconde et structure informationnelle: la relative de perception comme construction présentative, Langue française, 127, p. 49-66.

LEEMAN D., 2001, J'ai trouvé Kerstin qui travaillait: questions et hypothèse sur la relative prédicat de l'objet, in H. Kronning, C. Norén, B. Novén, G. Ransbo, L.G. Sundell, B. Svane (eds): Langage et référence, Mélanges offerts à Kerstin Jonasson, Uppsala : Acta Univ. Upsaliensis, p. 374-383.

MULLER C., 1995, Les relatives de perception: J'entends le garçon qui bégaie qui bégaie, in H. Bat-ZéevShyldkrot \& L. Kupferman (eds): tendances récentes en linguistique française et générale, volume dédié à David Gaatone, Amsterdam : Benjamins, p. 310-322.

MULler C., 1996, La subordination en français, Paris : Armand Colin.

MULLER C., 1998, Prédicats et prédication : quelques réflexions sur les bases de l'assertion, in M. Forsgren, K. Jonasson \& H. Kronning (eds) : Prédication, assertion, information, Actes du colloque d'Uppsala, Uppsala : Acta Univ. Upsaliensis, p. 355-366.

MULLER C., 2000, Les constructions à adjectif attribut de l'objet, entre prédication seconde et complémentation verbale, Langue française, 127, p. 21-35.

Muller C., 2002, Les bases de la syntaxe, Bordeaux : Presses Universitaires de Bordeaux (2e éd. : 2008). 
MULLER C, 2008, La relation au verbe principal dans les relatives prédicatives en français, in Faits de langue, $\mathrm{n}^{\circ}$ 31-32, La prédication, p. 337-346.

OLSSON K. 1976, La construction verbe + objet direct + complément prédicatif en français, Thèse de doctorat, Université de Stockholm.

PREBENSEN H., 1982, La proposition relative dite attributive, Revue Romane, 17-1, p. 98-117.

RothenBerg M., 1979, Les propositions relatives prédicatives et attributives: problème de linguistique française, $B S L, 74$, p. 351-395.

SCHWARZE C., 1974, Les constructions du type Je le vois qui arrive, in C.Rohrer, N.Ruwet (eds), Actes du Colloque Franco-allemand de Grammaire Transformationnelle, Etudes de Syntaxe, Tübingen : Niemeyer, $\mathrm{p}$. 18-30.

Résumé.

On examine les relatives prédicatives selon leurs propriétés distributionnelles. A côté des relatives liées à des verbes de perception sensorielle, on constate qu'il existe une construction proche qui est rendue possible par l'interprétation du verbe principal comme une mise en contact de son sujet avec le sujet sémantique de la relative. Dans les deux constructions, la relative a des propriétés aspectuelles verbales signifiant une action en cours. Au-delà, la relative prédicative peut encore apparaitre avec un statut différent, celui de "propriété ", basé sur les affinités des relatives avec les adjectifs attributs de prédication seconde. Il en existe deux catégories, d'une part des propriétés transitoires, et d'autre part des propriétés liees à une classe d'objets.

mots-clés : relative, prédicatif, événement, propriété, prédication seconde.

Abstract.

We examine the distribution of a very idiomatic construction: The French predicative relative, analysed as a verbal tensed predicate lacking a predicational subject. We show that its integration in the sentence is made possible by the interaction with the completed verb either as an associated event, or as a permanent or variable property. With perception verbs, and their extension to verbs describing a contact between the agent of the main clause and the one of the relative clause, the relative has verbal properties and describes an event. With some other verbs, the relative may occur, but as a property rather than an event, this being possible due to the adjectival properties of the relative clause.

key-words: relative, predicative, event, property, secondary predication.

Titre en anglais: Semantic interpretations and distribution of French predicative relatives. 